\title{
Composition of Lipopolysaccharide from Strains of Pseudomonas syringae pv. morsprunorum of Differing Host Specificity and Virulence
}

\author{
By SUSANNE E. ZAMZE, ${ }^{\dagger} \uparrow$ A. R. W. SMITH ${ }^{1 *}$ AND R. C. HIGNETT ${ }^{2}$ \\ ${ }^{1}$ School of Biological Sciences and Environmental Health, Thames Polytechnic, Wellington Street, \\ London SE18 6PF, UK \\ ${ }^{2}$ East Malling Research Station, East Malling, Maidstone, Kent ME19 6BJ, UK
}

(Received 15 November 1984 ; revised 13 February 1985)

\begin{abstract}
Lipopolysaccharide (LPS) from strains of Pseudomonas syringae pv. morsprunorum isolated from plum and cherry contained a uniform core region, which, in all cases, was composed of 2-keto-3deoxyoctonic acid, heptose, glucosamine, galactosamine, rhamnose, glucose, alanine, ethanolamine and phosphate. Lipid A contained glucosamine, phosphate and the fatty acids 3-OH $10: 0,12: 0,2-\mathrm{OH} 12: 0$, and 3-OH 12:0. Sidechains of LPS from two virulent plum isolates were composed of glucose and rhamnose, and were susceptible to hydrolysis by rhamnanase from the typing phage A7, which uses LPS as its binding site. Phage A7-resistant mutants of the virulent cherry isolate $\mathrm{C} 28$ contained rough LPS, did not adsorb A7, and displayed reduced virulence towards cherry. An avirulent, phage A7-resistant mutant of a plum isolate contained smooth LPS with sidechains of a high glucose content that were resistant to phage hydrolysis. Loss or alteration of sidechains therefore correlated with loss of virulence.
\end{abstract}

\section{INTRODUCTION}

Pseudomonas syringae pv. morsprunorum causes bacterial canker of Prunus avium (cherry) and Prunus domesticum (plum) trees in England (Garrett et al., 1966). The isolates from cherry and plum behave identically in biochemical tests but show unique specificity for a number of bacteriophages. Phage typing has demonstrated that, in the field, isolates from cherry and plum show a high degree of specificity for their homologous hosts. In field surveys, Crosse \& Garrett $(1963,1970)$ found that all their cherry isolates were sensitive to bacteriophage A7 and its related group of phages, whereas plum isolates, with only two known exceptions, were resistant. This resistance was not due to failure to adsorb the phage because adsorption occurred with equal efficiency to both cherry and plum strains (Garrett et al., 1974). Surface receptor sites for phage A7 are therefore present in both cherry and plum isolates.

Freigoun \& Crosse (1975) isolated variant cherry strains, which they designated race 2 , that differed in a number of physiological and pathological characters from the original (race 1) cherry isolates. Of 107 race 2 isolates examined, all adsorbed A7, but nearly one-third (34) were resistant to the phage. Garrett et al. (1974) observed that mutants of race 1 cherry isolates resistant to phage $\mathrm{A} 7$ were unable to adsorb the phage, and were always less virulent towards cherry. Alterations in the receptor site for phage A7 are therefore already known to affect virulence towards the plant.

The phage receptor site was identified by Quirk et al. (1976) as lipopolysaccharide (LPS). In a previous paper, (Smith et al., 1985), we described the structure of LPS from strain C28, a

† Present address: Infectious Diseases Unit, Department of Paediatrics, John Radcliffe Hospital, Headington, Oxford OX3 9DU, UK.

Abbreviations: KDO, 2-keto-3-deoxyoctonic acid; NGA, nutrient glycerol agar; PCP, phenol/chloroform/light petroleum extraction mixture. 
virulent, race 1 cherry isolate, detailing the composition of the lipid $\mathrm{A}$ and core regions, and showing that the sidechain is composed of simple rhamnan. Evidence was also presented that phage A7 possesses a rhamnanase (EC3.2.1.-) that hydrolyses the sidechains, releasing them as oligosaccharide.

It seemed possible to us that the resistance of plum isolates to lysis could be due to differences in the structure of the LPS, which blocked the progress of phage infection in these strains. The same structural differences might also be responsible for, or contribute to, the preference of the bacterial pathogen for its specific plant host.

The correlation of phage sensitivity with host specificity and virulence among plum and race 1 cherry isolates has prompted us to examine LPS from strains of $P$. syringae pv. morsprunorum to determine whether structural differences are discernible between the lipopolysaccharides of the two types of isolate, and what changes occur in LPS structure when race 1 cherry isolates mutate to phage A7-resistance with loss of virulence.

In the present paper we describe the composition of LPS from two virulent plum isolates and three avirulent, phage A7-resistant mutants. A preliminary report of the work was given by Zamze et al. (1981).

\section{METHODS}

Organisms. The following strains of $P$. syringae pv. morsprunorum were provided by Dr C. M. E. Garrett (East Malling Research Station): C28, a typical virulent, race 1 cherry isolate; D10, a typical virulent plum isolate; D17, an atypical virulent plum isolate, sensitive to phage A7 (Garrett \& Crosse, 1963) and D17-A1R, a mutant of D17, resistant to A7 and of reduced virulence (Garrett et al., 1974). Strains C28-1 and C28-2 were phage A7-resistant mutants of $\mathrm{C} 28$ isolated during the investigation reported here. Bacteria were grown and maintained as described by Smith et al. (1985). Bacteriophages were all obtained from Dr C. M. E. Garrett; their propagation and maintenance were also as described by Smith et al. (1985).

Phage experiments. Phage assays, sensitivity tests and adsorption tests were done as reported by Crosse \& Garrett (1963) and Garrett et al. (1974). In vitro inactivation of phage A7 with purified LPS was as described by Quirk et al. (1976).

Strain C28 was the indicator bacterium for all assays, except when testing for adsorption of A7 to strains D17 and D17-A1R, which are lysogenic (Garrett \& Crosse, 1963), releasing the phage L201 lytic for strain C28. In these cases strain $\mathrm{C} 12$, sensitive to $\mathrm{A} 7$ but resistant to $\mathrm{L} 201$, was the indicator.

Isolation and characterization of mutants of strain C28 resistant to phage A7. Portions (12 ml) of molten peptone/ glycerol/yeast extract (PGY) agar (Garrett et al., 1966), at $48^{\circ} \mathrm{C}$, were each inoculated with a suspension of strain C28 containing approximately $10^{10} \mathrm{c}$.f.u. and dispensed into Petri dishes. The set plates were each spread with $0.5 \mathrm{ml}$ phage $\mathrm{A} 7$ suspension containing $10^{9}$ p.f.u. and incubated at $18{ }^{\circ} \mathrm{C}$ for $4-5 \mathrm{~d}$. Mutants appeared as single colonies growing in areas of confluent lysis. These were picked off and purified by plating successively six times on nutrient glycerol agar (NGA; Hignett \& Quirk, 1979). Six phage resistant mutants, designated C28-1 to C28-6, were isolated; two, C28-1 and C28-2, were chosen at random for further study.

The identity of mutants was confirmed by: (a) their reaction in routine biochemical tests (Garrett et al., 1966); (b) comparison of their plasmid content with that of the parent strain (Errington, 1981), by the technique of single colony lysis (Eckhardt, 1978); (c) their sensitivity to phages other than A7; $(d)$ their ability to produce a hypersensitive response in tobacco (Klement, 1963). Their virulence compared with that of strain C28 was assessed by measuring their ability to initiate infections via leaf scars in the susceptible cherry cultivar Napoleon (Crosse \& Garrett, 1966). Inoculations were made in October on potted trees kept in the greenhouse. Inocula [10 $0^{8}$ $10^{9} \mathrm{c.f.u}$. ( $\mathrm{ml}$ distilled water $)^{-1}$ ] were applied to nine trees, each strain being inoculated onto ten leaf scars on one fruiting spur of each tree; the control treatment was sterile distilled water. Infections were scored in May of the following year.

Bulk culture of bacteria and extraction of LPS. Bacteria were grown in modified basal salts medium in 1 litre Erlenmeyer flasks as described previously (Smith et al., 1985). Larger quantities (8 litres) of strain C28 were cultured in the 10 litre baffled vessel of a Type 1/1000 LHE fermenter (LH Engineering Ltd, Farnham Common). The initial agitation and aeration rates were 750 r.p.m. and 2 litres air $\mathrm{min}^{-1}$ respectively. These were raised to 900 r.p.m. and 5 litres $\mathrm{min}^{-1}$ after approximately $30 \mathrm{~h}$ growth. The temperature was maintained at $18{ }^{\circ} \mathrm{C}$, and the $\mathrm{pH}$ at $6 \cdot 5$, by automatic addition of $0.5 \mathrm{M}-\mathrm{Na}_{2} \mathrm{CO}_{3}$. Cells were harvested after $40 \mathrm{~h}$ growth.

LPS was extracted from acetone-dried cells of strains C28, D10, D17 and D17-A1R with hot aqueous phenol according to the method of Westphal \& Jann (1965), and was purified as described by Smith et al. (1985). LPS was extracted from acetone-dried cells of strain C28-1 with phenol/chloroform/light petroleum, b.p. $40-60{ }^{\circ} \mathrm{C}(\mathrm{PCP})$ according to the method of Galanos et al. (1969). In order to extract LPS from strain C28-2 this procedure was modified as follows. Freeze-dried cells were homogenized with PCP at room temperature, [4 ml PCP $\left.(\mathrm{g} \mathrm{cells})^{-1}\right]$. 
The mixture was centrifuged at $5000 \mathrm{~g}$ for $15 \mathrm{~min}$ and the supernatant retained. The extraction was repeated and the second supernatant combined with the first. Light petroleum and chloroform were removed by rotary evaporation in vacuo at $40^{\circ} \mathrm{C}$ and an equal volume of distilled water was added to the remaining phenol solution. The mixture was dialysed exhaustively against distilled water and extracted twice with 2 to 3 volumes of chloroform to remove lipid.

Samples of LPS from C28-1 and C28-2 were finally deposited by ultracentrifugation at $100000 \mathrm{~g}$ for $3 \mathrm{~h}$ at $4{ }^{\circ} \mathrm{C}$, resuspended in distilled water and treated with ribonuclease for $18 \mathrm{~h}$ at $4{ }^{\circ} \mathrm{C}$. Ultracentrifugation and extraction with chloroform were repeated, and the LPS preparations stored at $4^{\circ} \mathrm{C}$ over chloroform.

Action of phage A7 rhamnanase on LPS. Washed particles of phage A7 $\left(3 \times 10^{12}\right.$ p.f.u.) were incubated with LPS $(3 \mathrm{mg})$ in distilled water $(2 \mathrm{ml})$ at room temperature for $1 \mathrm{~h}$, with occasional shaking. The products were separated on a small column $(0.6 \times 20 \mathrm{~cm})$ of Sepharose $4 \mathrm{~B}$ eluted with distilled water at a flow rate of $0 \cdot 25 \mathrm{ml} \mathrm{min}^{-1}$. Fractions $(0.5 \mathrm{ml})$ were assayed for total carbohydrate with phenol/sulphuric acid reagent (Dubois et al., 1956).

Chromatography and analytical methods. Descending paper chromatography was done on Whatman no. 1 paper using the following solvent systems: A, butan-1-ol/pyridine/water $(6: 4: 3$, by vol.); B, butan-1-ol/acetic acid/water $(12: 3: 5$, by vol.); C, the upper phase of butan-1-ol/ethanol/water/aqueous ammonia (sp. gr. 0.88) $(40: 10: 49: 1$, by vol.). Detection reagents were the same as those used previously (Smith et al., 1985).

The procedures for the hydrolysis and chemical analysis of LPS, for gel permeation chromatography, and for the auto-analysis of amino compounds were the same as those used by Smith et al. (1985).

Fatty acids were analysed by GLC as the methyl esters after their liberation from LPS (1-5 mg) (SalkinojaSalonen \& Boeck, 1978). Analysis was done on a column $(3.2 \times 183 \mathrm{~mm})$ of $3 \%$ SP2100-DOH on $100 / 120 \mathrm{mesh}$ Supelcoport (Supelco), operated with temperature programming from $150-225{ }^{\circ} \mathrm{C}$ at $2{ }^{\circ} \mathrm{C} \min ^{-1}$ and $\mathrm{N}_{2}$ $\left(20 \mathrm{ml} \mathrm{min}^{-1}\right)$ as the carrier; flame ionization detection was used. Results were quantified by integration of peak areas, taking the response factor to be 1 on a weight basis in all cases (Rietschel et al., 1972).

\section{RESULTS}

\section{Characterization of phage A7-resistant mutants of $C 28$}

Unlike the parent strain $\mathrm{C} 28$, which adsorbed $98 \%$ of the phage particles under the conditions of the test, mutants C28-1 to C28-6 all failed to adsorb A7. All six mutants had also acquired resistance to two other phages, A9 and A44, that are specific for cherry strains of $P$. syringae pv. morsprunorum, but they remained sensitive to phages A15, A26 and S6, which lyse both cherry and plum strains of pv. morsprunorum and strains of pv. syringae. Like C28, they were also resistant to phage $\mathrm{B} 1$, the typing phage for race 2 isolates of pv. morsprunorum (Persley \& Crosse, 1978).

The mutants all behaved as race 1 strains of $P$. syringae pv. morsprunorum in biochemical tests (Garrett et al., 1966), and induced the hypersensitive response in tobacco. On NGA, all six mutants produced flat colonies with wavy, irregular margins, whereas the parent strain produced convex colonies with entire edges (Fig. 1). As with the parent strain, growth of the
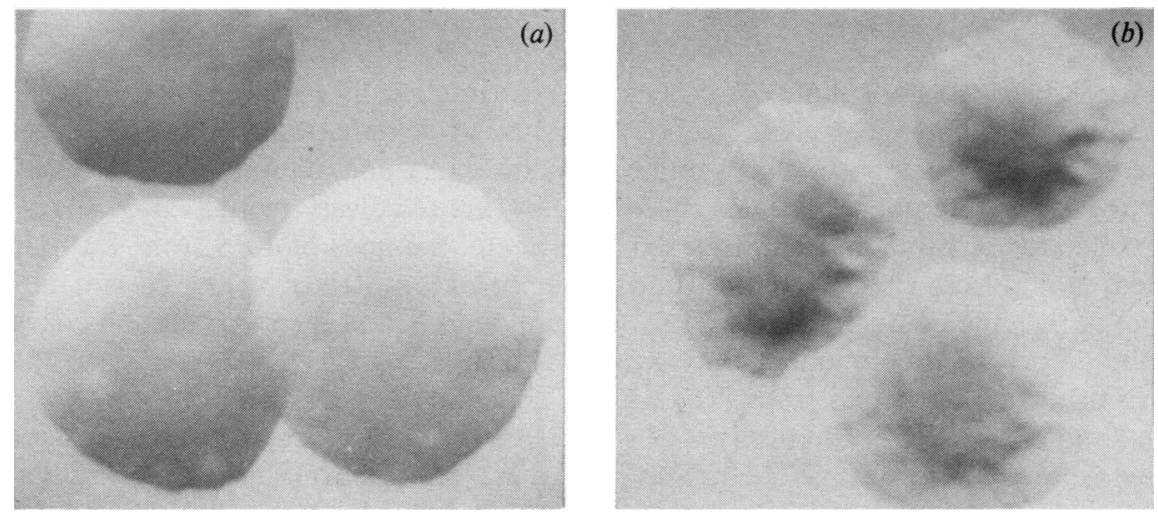

Fig. 1. Colonies of $P$. syringae pv. morsprunorum $\mathrm{C} 28(a)$ and the mutant $\mathrm{C} 28-1(b)$ on glycerol/nutrient agar. Grown at $25^{\circ} \mathrm{C}$ for $5 \mathrm{~d}$. 
Table 1. Virulence of $P$. syringae pv. morsprunorum strain $C 28$ and phage resistant mutants $C 28-1$ and C28-2 towards cherry $c v$. Napoleon

Each strain was inoculated onto a total of 90 leaf scars on 9 trees as detailed in Methods. Inoculations were made in October and the trees were examined for necrotic buds and the development of cankers during the following May. Results for buds are expressed as percentages of the total number of leaf scars inoculated.

\begin{tabular}{|c|c|c|c|c|}
\hline Strain & $\begin{array}{c}\text { Necrotic } \\
\text { buds } \\
(\%)\end{array}$ & $\begin{array}{c}\text { Buds showing } \\
\text { delayed and } \\
\text { poor development } \\
(\%)\end{array}$ & $\begin{array}{c}\text { Total buds } \\
\text { showing } \\
\text { infection } \\
(\%)\end{array}$ & $\begin{array}{c}\text { Number } \\
\text { of } \\
\text { cankers }\end{array}$ \\
\hline $\mathrm{C} 28$ & 46 & 35 & 81 & 3 \\
\hline C28-1 & 16 & 31 & 47 & 1 \\
\hline C $28-2$ & 17 & 26 & 43 & 1 \\
\hline Control & 0 & 9 & 9 & 0 \\
\hline
\end{tabular}

mutants on sucrose nutrient agar (Garrett et al., 1966) led to the synthesis of levan and the production of large domed colonies.

Errington (1981) demonstrated, by agarose-gel electrophoresis of single colony lysates, that strain C28 contains four plasmids of molecular weight $88,52,25$, and $22 \times 10^{6}$, respectively. Four plasmids with identical molecular weights to these were found in each of the mutants C28-1 to C28-6, and in the parent strain, during this study.

Results of the virulence tests on strains C28, C28-1 and C28-2 in leaf scar inoculations of cv. Napoleon are shown in Table 1. Both C28-1 and C28-2 had reduced virulence compared with C28.

\section{Extraction and purity of LPS}

Yields of LPS extracted with hot aqueous phenol from acetone-dried cells of strains D10, D17 and D17-A1R were $3 \cdot 6,3.3$ and $1.5 \%(w / w)$, respectively. It was not possible to extract the LPS with hot aqueous phenol from either C28-1 or C28-2. Extraction with PCP, however, yielded LPS from acetone-dried cells of strain C28-1, with a yield of $2.5 \%(\mathrm{w} / \mathrm{w})$. Using a modification of the PCP method, LPS could also be extracted from freeze-dried cells of strain C28-2, with a yield of $1.7 \%(w / w)$.

After purification, all LPS samples contained less than $0.5 \%(\mathrm{w} / \mathrm{w})$ of nucleic acid. Samples of LPS from D10, D17 and D17-A1R contained $1-2 \%(\mathrm{w} / \mathrm{w})$ protein as estimated with Folin's reagent. Auto-analysis revealed small amounts of the common amino acids which were thought to be derived from the hydrolysis of the residual protein, whereas LPS samples from C28-1 and C28-2 were entirely free of protein. Neither diaminopimelic acid nor muramic acid was detected by auto-analysis, indicating that samples were free of peptidoglycan.

\section{Analysis of whole LPS}

Heptose, KDO and phosphate were detected colorimetrically in the LPS from all strains. Paper chromatography of hydrolysates of the polysaccharide moieties of each LPS revealed the presence of glucose, rhamnose, glucosamine, galactosamine and alanine. The presence of the latter three amino components was confirmed by auto-analysis, which also revealed the presence of ethanolamine and amino sugar phosphate. Samples of LPS from D10 and D17 contained, in addition, small amounts of an unidentified amino-compound, $X$, which totalled $0.04 \mu \mathrm{mol}$ norleucine equivalents (mg LPS) ${ }^{-1}$. Compound $\mathrm{X}$ had the same elution characteristics as the unidentified component previously found in C28 LPS (Smith et al., 1985). It was absent from the LPS samples from D17-A1R, C28-1 and C28-2.

Table 2 shows the overall composition of whole LPS from the different strains. The results of the analysis of LPS from strain C28, the subject of a previous report (Smith et al., 1985), and the strain from which C28-1 and C28-2 were derived, are included for comparison. The percentage weight of lipid A and the amounts of KDO, heptose, glucosamine, galactosamine, alanine and phosphate were similar in LPS isolated from strains D10, D17 and D17-A1R, and C28. 
Table 2. Composition of LPS from strains of P. syringae pv. morsprunorum

\begin{tabular}{|c|c|c|c|c|c|c|}
\hline Component & D10 & D17 & D17-A1R & C28* & C28-1 & C28-2 \\
\hline KDO & $0 \cdot 12$ & 0.09 & 0.09 & $0 \cdot 10$ & $0 \cdot 14$ & 0.07 \\
\hline Total carbohydrate & $2 \cdot 30$ & $2 \cdot 70$ & $2 \cdot 90$ & $2 \cdot 30$ & $0 \cdot 88$ & $0 \cdot 70$ \\
\hline Rhamnose & $1 \cdot 50$ & $1 \cdot 54$ & 0.31 & 1.90 & $0 \cdot 17$ & 0.20 \\
\hline Heptose & 0.06 & 0.06 & 0.06 & 0.07 & $0 \cdot 12$ & $0 \cdot 26$ \\
\hline Glucose & 0.37 & 0.56 & $1 \cdot 41$ & $0 \cdot 25$ & $0 \cdot 30$ & $0 \cdot 30$ \\
\hline Phosphate & $1 \cdot 19$ & $1 \cdot 12$ & $1 \cdot 07$ & $1 \cdot 38$ & $1 \cdot 80$ & $2 \cdot 30$ \\
\hline $\mathrm{Ca}^{2+}$ & 0.31 & 0.29 & $0 \cdot 25$ & 0.48 & $0 \cdot 11$ & 0.24 \\
\hline $\mathrm{Mg}^{2+}$ & $0 \cdot 32$ & $0 \cdot 30$ & $0 \cdot 13$ & 0.20 & 0.68 & $0 \cdot 20$ \\
\hline $\mathrm{K}^{+}$ & 0.05 & 0.03 & $0 \cdot 001$ & $0 \cdot 004$ & 0.03 & 0.09 \\
\hline \multicolumn{7}{|l|}{$\begin{array}{c}\text { Amino compounds in } \\
\text { whole LPS: }\end{array}$} \\
\hline Glucosamine & $0 \cdot 39$ & + & $0 \cdot 26$ & $0 \cdot 38$ & + & + \\
\hline Galactosamine & $0 \cdot 14$ & + & $0 \cdot 12$ & $0 \cdot 17$ & + & + \\
\hline Amino sugar phosphate & 0.02 & + & 0.06 & 0.08 & + & + \\
\hline Ethanolamine & 0.09 & + & 0.05 & 0.06 & + & + \\
\hline Alanine & $0 \cdot 13$ & + & $0 \cdot 16$ & $0 \cdot 19$ & + & + \\
\hline \multicolumn{7}{|l|}{$\begin{array}{l}\text { Amino compounds in the } \\
\text { polysaccharide moiety: }\end{array}$} \\
\hline Hexosamine & $0 \cdot 13$ & $0 \cdot 12$ & $0 \cdot 15$ & $0 \cdot 19$ & $0 \cdot 19$ & $0 \cdot 16$ \\
\hline Total amino compounds & 0.50 & $0 \cdot 26$ & 0.65 & 0.58 & 0.76 & $0 \cdot 60$ \\
\hline Lipid A† & 24 & 28 & 29 & 29 & 33 & 40 \\
\hline \multicolumn{7}{|c|}{$\begin{array}{l}+ \text {, Components present but not quantified. } \\
{ }^{*} \text { Results of Smith et al. }(1985) \text {. } \\
\dagger \text { Results expressed as } \%(w / w)\end{array}$} \\
\hline
\end{tabular}

Rhamnose and glucose, however, were present in differing proportions. In comparison with C28, samples of LPS from D10, D17 and D17-A1R contained more glucose and less rhamnose. In particular, LPS from D17-A1R differed from C28 LPS in containing approximately six times as much glucose but only one-third the amount of rhamnose. All of the above components were also found in LPS from the phage A7-resistant mutants, C28-1 and C28-2, but in different amounts from those in C28 LPS. The percentage weight of lipid A was higher and the rhamnose content was markedly decreased. Both contained approximately one-tenth the amount of rhamnose. The relative amounts of KDO, glucose, phosphate and amino compounds in the LPS from C28-1 were slightly higher than in LPS from C28, and the relative amount of heptose was approximately doubled; the LPS from C28-2 contained a greater proportion of glucose and phosphate than C28 LPS and four times as much heptose. No increase, however, was detected in the relative content of KDO and amino compounds in C28-2 LPS. The KDO: heptose ratio in LPS from C28-1 and C28-2 was $1 \cdot 2: 1$ and $0 \cdot 25: 1$ respectively, compared with a ratio of $1 \cdot 5: 1$ in C28 LPS. Samples of LPS from all strains contained appreciable quantities of $\mathbf{M g}^{2+}$ and $\mathrm{Ca}^{2+}$. In particular, C28-1 LPS contained a large amount of $\mathbf{M g}^{2+}$. Very little $\mathrm{K}^{+}$was detected, except in LPS from C28-1, in which the molar ratio of $\mathrm{Mg}^{2+}: \mathrm{K}^{+}$was $2: 1$. The combined molar content of the cations $\mathrm{Mg}^{2+}, \mathrm{Ca}^{2+}$ and $\mathrm{K}^{+}$was $0.68,0.62,0.32,0.83$ and $0.57 \mu \mathrm{mol}$ per $\mathrm{mg}$ LPS isolated from strains D10, D17, D17-A1R, C28-1 and C28-2 respectively, compared with $0.68 \mu \mathrm{mol}(\mathrm{mg}$ C28 LPS) ${ }^{-1}$. The molar ratio of $\mathrm{Ca}^{2+}: \mathrm{Mg}^{2+}$ was $2: 1$ in LPS from D17-A1R, $1: 1$ in LPS from D10, D17 and C28-2, and 1:6 in LPS from C28-1.

\section{Composition of lipid $\boldsymbol{A}$}

Paper chromatography revealed the presence of glucosamine together with trace amounts of galactosamine and alanine in hydrolysates of lipid A from each strain. Lipid A from each strain also contained approximately $15 \%(\mathrm{w} / \mathrm{w})$ of the total phosphate content of the LPS. Each LPS 
contained the same major fatty acids, namely 3-OH $10: 0,12: 0,2-\mathrm{OH} 12: 0$ and $3-\mathrm{OH} 12: 0$, together with trace amounts of 16:0 (Table 3). Samples of LPS from C28-1 and C28-2 also contained small amounts of longer chain fatty acids which were tentatively identified as a-17:0 and 18:0. These were probably contaminants that arose from the methanolysis of lipids coextracted with LPS by the PCP mixture.

\section{Partial hydrolysis and fractionation of LPS}

Mild acid hydrolysis of LPS with $0.01 \mathrm{M}-\mathrm{HCl}$ (Smith et al., 1985) was used to release lipid A whilst leaving the core and sidechain regions intact. The first precipitate of lipid A from the LPS of D10, D17 and D17-A1R appeared after hydrolysis for $40 \mathrm{~min}$ at $100^{\circ} \mathrm{C}$. Hydrolysis was allowed to continue for a further $20 \mathrm{~min}$, by which time release of lipid $\mathrm{A}$ was judged to be complete from the clarity of the supernatant. In contrast, lipid A first precipitated from the LPS of C28-1 and C28-2 after $20 \mathrm{~min}$, and precipitation was complete after a further $20 \mathrm{~min}$.

The partially hydrolysed polysaccharide moieties from each LPS were separated by gel permeation chromatography on columns of Sephadex G-50. LPS from strains D10, D17 and D17-A1R all had similar profiles, with three peaks. The high molecular weight peak (I) emerged with the void volume of the column and contained carbohydrate with little or no phosphate. The intermediate peak (II) contained both components. The low molecular weight peak (III), which emerged with the totally included volume, contained phosphate but very little carbohydrate.

In contrast, LPS from C28-1 and C28-2 produced only two peaks. These contained both carbohydrate and phosphate, and eluted at positions equivalent to those of the lower molecular weight peaks (II and III) derived from LPS of strains D10, D17 and D17-A1R, which were also equivalent to the peaks II and III derived from the LPS of strain C28. Fractions containing peak material were pooled for further analysis; the results are given in Table 4.

By analogy with previous findings for C28 LPS (Smith et al., 1985), it is proposed that peak I represented the complete polysaccharide (sidechain and core), that peak II represented the core oligosaccharide and that peak III represented the degradation products of the linkage region of intact LPS. Sidechains from D10, D17 and D17-A1R thus contained both rhamnose and glucose in the ratios $9 \cdot 6,2 \cdot 6$ and $0 \cdot 2: 1$ respectively.

The components found in each of the peaks II and III were similar in LPS obtained from D10, D17 and D17-A1R. The core peak II contained heptose, glucose, rhamnose, alanine, phosphate, amino sugars and a small amount of KDO. Peak III contained KDO, phosphate, amino compounds (including small amounts of amino sugar), and small quantities of glucose and rhamnose. A constant proportion ( $70 \%$ ) of the total phosphate found in peak III was present in the free form, i.e. oxidation of samples was not required for its detection; some phosphate had, therefore, presumably been released from the LPS during mild acid hydrolysis. Peaks II and III from C28-1 LPS were very similar in composition to those derived from the samples of smooth LPS.

\section{Interaction of phage $A 7$ with LPS}

Cells of strains D10 and D17 adsorbed $98 \%$ of phage A7 under the conditions employed whereas adsorption of A7 by strains D17-A1R, C28-1 and C28-2 was zero. Inactivation of A7 by LPS in vitro was measured as the percentage decrease in p.f.u. after incubation of mixtures of LPS and phage suspension containing $10^{4}$ p.f.u. $\mathrm{ml}^{-1}$ at room temperature for $1 \mathrm{~h}$. Samples of LPS from D10 and D17 $\left(50 \mu \mathrm{g} \mathrm{ml}^{-1}\right)$ inactivated $98 \%$ of phage A7 whereas LPS from D17-A1R, at the same concentration, inactivated only $27 \%$ of the phage. Incubation with LPS from C28-1 and C28-2 at concentrations as high as $500 \mu \mathrm{g} \mathrm{LPS} \mathrm{m}^{-1}$ resulted in zero inactivation.

To determine whether phage A7 hydrolysed the sidechains of the LPS from plum strains, washed phage particles were incubated with LPS in distilled water, essentially as described by Smith et al. (1985), and the products were rapidly separated on a small column of Sepharose 4B. The LPS of D10 and D17 both yielded two carbohydrate peaks. The first emerged with the void volume and resembled LPS in giving pronounced light scatter. The second peak was totally included, and on further analysis was shown to contain only rhamnose and glucose in the same ratio as found in the sidechain material derived from LPS by mild acid hydrolysis. The products 


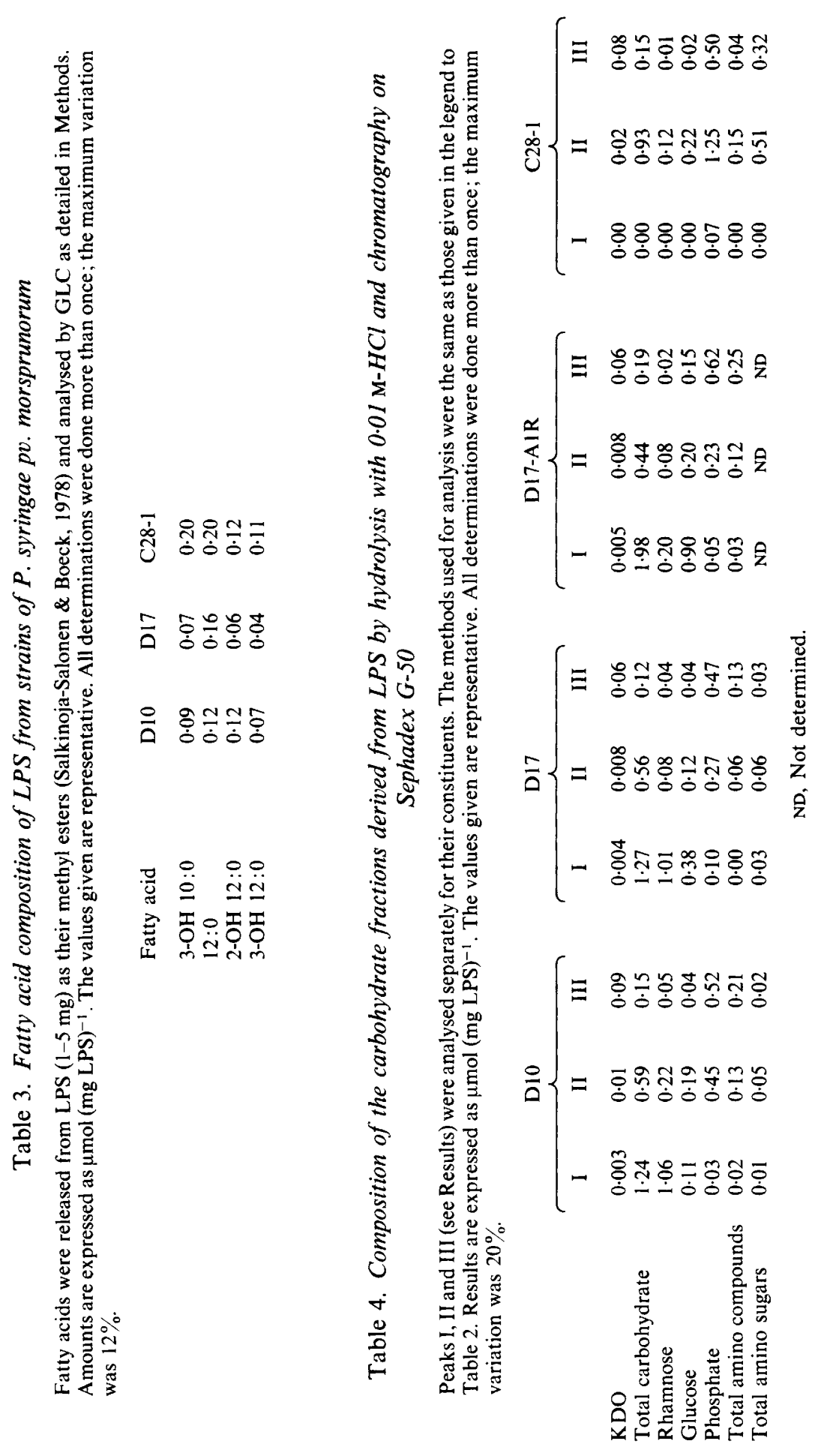


of incubation from D17-A1R emerged almost entirely with the void volume. The small amount of material which was totally included consisted mostly of glucose together with a trace of rhamnose. It was concluded, therefore, that the sidechains from D10 and D17 were susceptible to hydrolysis by A7 but that those from D17-A1R were resistant.

\section{DISCUSSION}

The composition of the core regions of LPS from the different plum strains was very similar, and closely resembled that of the cherry strain C28 (Smith et al., 1985), indicating similar if not identical structures for this region in all four strains. Differences between LPS samples were found in the sidechain regions. In contrast to the rhamnan sidechain of $\mathrm{C} 28$, sidechains from the plum strains contained differing amounts of rhamnose and glucose. The quantity of rhamnose in the sidechains from D10 and D17 was approximately the same as that in C28, but the sidechains from D17-A1 R contained only one quarter of this amount together with a much greater quantity of glucose. Thus glucose had replaced rhamnose as the major sidechain component in this LPS.

Sidechains from the plum strain LPS were, therefore, apparently heteropolysaccharides. It is also conceivable, however, that the LPS existed entirely as two separate molecular species, one containing rhamnan sidechains and the other glucan sidechains. Different proportions of molecular types occurring in each strain would account for the different ratios of rhamnose and glucose observed.

The phage-A7 resistant mutants, C28-1 and C28-2, were shown to elaborate a rough type of LPS that entirely lacked sidechain material but was thought to contain complete core regions. The polysaccharide moieties of both C28-1 and C28-2 LPS were quantitatively similar to the core oligosaccharide of LPS from the parent strain C28 (Smith et al., 1985). As expected for rough type LPS, and in comparison with LPS from C28, there was a small increase in the proportion of most of the core components relative to the total weight of LPS. There was, however, a large increase in the amount of heptose detected, especially in LPS from C28-2, but the amounts of KDO and amino compounds detected in C28-2 LPS were, on a weight basis, the same as in C28 LPS. The properties of the rough LPS showed a number of differences compared with smooth LPS: they were noticeably less soluble in water, adsorbed to Millipore filters, and migrated in $1 \%$ agarose during electrophoresis, the latter two steps being routinely used for the purification of smooth LPS. The samples of rough LPS were also less stable to hydrolysis with mild acid.

The composition of lipid A was the same in LPS from each of the plum strains and was also identical with that of $\mathrm{C} 28$. Some differences in the amounts of the individual fatty acids were detected between strains; the total percentage weight of fatty acids recovered from D10 and D17 was also less than from C28. As would be expected for rough strains, LPS from C28-1 and C28-2 contained a higher percentage of lipid A by weight than did C28 LPS. The molar ratios of fatty acids recovered from C28-1, C28-2 and C28 LPS differed noticeably, particularly in respect of the relative quantity of $2-\mathrm{OH} 12: 0$.

Mutation resulting in the synthesis of a rough LPS is frequently encountered in species of Enterobacteriaceae (Lüderitz et al., 1971) but very few rough LPS mutants outside the Enterobacteriaceae have been described. Two such examples are mutants of Pseudomonas solanacearum (Whatley et al., 1980) and Pseudomonas aeruginosa (e.g. Koval \& Meadow, 1977; Jarrell \& Kropinski, 1977, 1981). Unlike P. syringae pv. morsprunorum, mutation to a rough LPS in $P$. aeruginosa was not always found to correspond with a switch to rough colonial morphology. Mutants of $P$. aeruginosa synthesizing LPS with various degrees of defect in the core region have been isolated and characterized (Koval \& Meadow, 1977; Rowe \& Meadow, 1983).

Adsorption of phage A7 to whole cells of $P$. syringae pv. morsprunorum correlated with the inactivation of the phage in vitro by purified LPS and with hydrolysis of the sidechains. Hydrolysis of the sidechains from the plum strains D10 and D17 occurred despite differences in their composition from those of strain C28. The occurrence of a small amount of glucose together with rhamnose in these polysaccharides does not appear to alter their capacity to act as receptors and substrates for $\mathrm{A} 7$. Infection of strain D10, and presumably other plum strains, 
would also appear to be blocked at a later stage of the process. In contrast, changes in the sidechain composition of D17-A1R were sufficient to prevent interaction of LPS from this organism with A7.

Loss of virulence in P. syringae pv. morsprunorum was found to be associated with changes in LPS structure - either modification of the sidechain polysaccharide (as in D17-A1R) or complete loss of sidechains to yield a rough LPS. Mutations in LPS are known, however, to have indirect effects on the structure and integrity of the outer membrane. For example, mutation to a rough LPS in the Enterobacteriaceae has been shown to result in an increased permeability of the outer membrane (e.g. Tamaki \& Matsuhashi, 1973; Sanderson et al., 1974), and a loss of heptose from the core region may also be associated with diminished quantities of protein in the outer membrane (e.g. Ames et al., 1974; Koplow \& Goldfine, 1974; Smit et al., 1975). It is possible that the potential of pv. morsprunorum to act as a pathogen is highly dependent on the integrity of its outer membrane, and that any one of a number of changes in this region could result in an impairment of virulence. Thus, avirulence in these mutants may not be a direct result of structural changes in their LPS per se but may, possibly, be due to such associated factors.

The involvement of extracellular polysaccharide in the pathogenicity of bacteria towards plants, and the association of loss of ability to produce polysaccharide with loss of virulence is well documented (e.g. Bradshaw-Rouse et al., 1981; Bennett \& Billing, 1978). P. syringae pv. morsprunorum is unusual in that, as far as is known, it produces extracellular polysaccharide only when grown in a nutrient sucrose medium. It may be postulated that an extensive sidechain polysaccharide region in this bacterium plays a similar role to an extracellular polysaccharide in determining its virulence. Findings with strain D17-A1R, which elaborates a modified sidechain, suggest that the structure of the sidechain is also important to virulence. Investigation of the role of LPS in the virulence of phytopathogens has, so far, been limited to only a few bacterium-plant associations, e.g. $P$. solanacearum and tobacco (Whatley et al., 1980), Xanthomonas phaseoli and soybean (Whatley \& Sequeira, 1981). In both cases a correlation between the production of a smooth LPS and virulence was found. A similar association was indicated in P. syringae pv. phaseolicola, where a decrease in the rhamnose content of the LPS was accompanied by a reduction in virulence towards bean (Anderson, 1980).

Susanne E. Zamze was supported by a SERC CASE Studentship. We also wish to thank Dr C. M. E. Garrett for source cultures; $\mathrm{Mr}$ T. Samuelson for atomic absorption and emission spectroscopy; Dr J. Errington for agarose gel electrophoresis of single colony lysates and $\mathrm{Mr} \mathrm{B}$. Eldridge for photography.

\section{REFERENCES}

Ames, G. F.-L., Spudich, E. N. \& Nikaido, H. (1974). Protein composition of the outer membrane of Salmonella typhimurium: effect of lipopolysaccharide mutations. Journal of Bacteriology 117, 406-416.

ANDERSON, A. J. (1980). Unique aspects of the cell surface polysaccharide of Pseudomonas phaseolicola as demonstrated by bacteriophage specificity. Canadian Journal of Microbiology 26, 1422-1427.

Bennetr, R. A. \& Billing, E. (1978). Capsulation and virulence in Erwinia amylovora. Annals of Applied Biology 89, 41-45.

Bradshaw-Rouse, J. J., Whatley, M. H., Coplin, D. L., Woods, A., Sequeira, L. \& Kelman, A. (1981). Agglutination of Erwinia stewartii strains with a corn agglutinin: correlation with extracellular polysaccharide production and pathogenicity. Applied and Environmental Microbiology 42, 344-350.

Crosse, J. E. \& Garrett, C. M. E. (1963). Studies on the bacteriophagy of Pseudomonas morsprunorum, Ps. syringae and related organisms. Journal of Applied Bacteriology 26, 159-177.
Crosse, J. E. \& Garrett, C. M. E. (1966). Bacterial canker of stone fruits. VII. Infection experiments with Pseudomonas morsprunorum and Ps. syringae. Annals of Applied Biology 58, 31-41.

Crosse, J. E. \& Garrett, C. M. E. (1970). Pathogenicity of Pseudomonas morsprunorum in relation to host specificity. Journal of General Microbiology 62, 315327.

Dubois, M., Gilles, K. A., Hamilton, J. K., Rebers, P. A. \& SMITH, F. (1956). Colorimetric method for determination of sugars and related substances. Analytical Chemistry 28, 350-356.

ECKHARDT, T. (1978). A rapid method for the identification of plasmid DNA in bacteria. Plasmid 1, 584-588.

Errington, J. (1981). The genetics of Pseudomonas morsprunorum. $\mathrm{PhD}$ thesis, Thames Polytechnic.

Freigoun, S. O. \& Crosse, J. E. (1975). Host relations and distribution of a physiological and pathological variant of Pseudomonas morsprunorum. Annals of Applied Biology 81, 317-330. 
Galanos, C., LÜDeritz, O. \& WestPhal, O. (1969). A new method for the extraction of R-lipopolysaccharides. European Journal of Biochemistry 9, 245-249.

Garrett, C. M. E. \& Crosse, J. E. (1963). Observations on lysogeny in the plant pathogens Pseudomonas morsprunorum and Ps. syringae. Journal of Applied Bacteriology 26, 27-34.

Garrett, C. M. E., Panagopoulos, C. G. \& Crosse, J. E. (1966). Comparison of plant pathogenic pseudomonads from fruit trees. Journal of Applied Bacteriology 29, 342-356.

Garrett, C. M. E., Crosse, J. E. \& Sletten, A. (1974). Relations between phage sensitivity and virulence in Pseudomonas morsprunorum. Journal of General Microbiology 80, 475-483.

Hignett, R. C. \& QUIRK, A. V. (1979). Properties of phytotoxic cell-wall components of plant-pathogenic pseudomonads. Journal of General Microbiology 110, $77-81$.

JARRELL, K. F. \& KROPINSKI, A. M. B. (1977). The chemical composition of the lipopolysaccharide from Pseudomonas aeruginosa strain PAO and a spontaneously derived rough mutant. Microbios 19, 103-116.

JARRELL, K. F. \& KROPINSKI, A. M. B. (1981). Pseudomonas aeruginosa bacteriophage $\phi$ PLS 27lipopolysaccharide interactions. Journal of Virology 40, 411-420.

KLEMENT, Z. (1963). Rapid detection of phytopathogenicity of phytopathogenic pseudomonads. Nature, London 199, 299-300.

Koplow, J. \& GoldFINE, H. (1974). Alterations in the outer membrane of the cell envelope of heptosedeficient mutants of Escherichia coli. Journal of Bacteriology 117, 527-543.

Koval, S. F. \& Meadow, P. M. (1977). The isolation and characterization of lipopolysaccharide-defective mutants of Pseudomonas aeruginosa PAC1. Journal of General Microbiology 98, 387-398.

LÜderitz, O., WestPhal, O., Staub, A. M. \& NIKAIDO, H. (1971). Isolation and chemical and immunological characterization of bacterial lipopolysaccharides. In Microbial Toxins, vol. IV, pp. 145-233. Edited by G. Weinbaum, S. Kadis \& S. J. Ajl. New York: Academic Press.

Persley, G. J. \& Crosse, J. E. (1978). A bacteriophage specific to race 2 of the cherry strains of Pseudomonas morsprunorum. Annals of Applied Biology 89, 219-222.

Quirk, A. V., Sletten, A. \& Hignett, R. C. (1976). Properties of phage receptor lipopolysaccharide from Pseudomonas morsprunorum. Journal of General Microbiology 96, 375-381.
RIeTSCHEL, E., GoTTERT, H., LÜDERITZ, O. \& WestPHAL, O. (1972). Nature and linkages of the fatty acids present in the lipid A component of Salmonella lipopolysaccharides. European Journal of Biochemistry 28, 166-173.

Rowe, P. S. N. \& MEADow, P. M. (1983). Structure of the core oligo-saccharide from the lipopolysaccharide of Pseudomonas aeruginosa PAC1R and its defective mutants. European Journal of Biochemistry 132, 329-337.

Salkinoja-SAlonen, M. \& Boeck, R. (1978). Characterization of lipopolysaccharides isolated from $\mathrm{Agro}$ bacterium tumefaciens. Journal of General Microbio$\log y$ 105, 119-125.

Sanderson, K. E., MacAlister, T., Costerton, J. W. \& CHENG, K. J. (1974). Permeability of lipopolysaccharide-deficient (rough) mutants of Salmonella typhimurium to antibiotics, lysozyme and other agents. Canadian Journal of Microbiology 20, 11351145 .

Smit, J., Kamio, Y. \& NiKaido, H. (1975). Outer membrane of Salmonella typhimurium: chemical analysis and freeze-fracture studies with lipopolysaccharide mutants. Journal of Bacteriology 124, 942958.

Smith, A. R. W., Zamze, S. E. \& Hignett, R. C. (1985). Composition of lipopolysaccharide from Pseudomonas syringae pv. morsprunorum and its digestion by bacteriophage A7. Journal of General Microbiology 131, 963-974.

TAMAKI, S. \& MatsuHaShI, M. (1973). Increase in sensitivity to antibiotics and lysozyme on deletion of lipopolysaccharides in Escherichia coli strains. Journal of Bacteriology 114, 453-454.

WestPhal, O. \& JANN, K. (1965). Bacterial lipopolysaccharides: extraction with phenol-water and further application of the procedures. Methods in Carbohydrate Chemistry 5, 83-91.

Whatley, M. H. \& Sequeira, L. (1981). Bacterial attachment to plant cell walls. Recent Advances in Phytochemistry 15, 213-240.

Whatley, M. H., Hunter, N., Cantrell, M. A., HeNDRICK, C., KeEgSTRA, K. \& SeQueIRA, L. (1980). Lipopolysaccharide composition of the wilt pathogen, Pseudomonas solanacearum. Correlation with the hypersensitive response in tobacco. Plant Physio$\log y$ 65, 557-559.

Zamze, S. E., Smith, A. R. W. \& Hignett, R. C. (1981). Composition of lipopolysaccharide from Pseudomonas morsprunorum strains of differing host specificity and virulence. Society for General Microbiology Quarterly 8, 262-263. 